\title{
Multiple Positive Solutions for a Coupled System of $p$-Laplacian Fractional Order Two-Point Boundary Value Problems
}

\author{
K. R. Prasad ${ }^{1}$ and B. M. B. Krushna ${ }^{2}$ \\ ${ }^{1}$ Department of Applied Mathematics, Andhra University, Visakhapatnam 530 003, India \\ ${ }^{2}$ Department of Mathematics, MVGR College of Engineering, Vizianagaram 535 005, India
}

Correspondence should be addressed to K. R. Prasad; rajendra92@rediffmail.com

Received 24 February 2014; Accepted 21 April 2014; Published 7 May 2014

Academic Editor: Bashir Ahmad

Copyright (c) 2014 K. R. Prasad and B. M. B. Krushna. This is an open access article distributed under the Creative Commons Attribution License, which permits unrestricted use, distribution, and reproduction in any medium, provided the original work is properly cited.

This paper establishes the existence of at least three positive solutions for a coupled system of $p$-Laplacian fractional order twopoint boundary value problems, $D_{0^{+}}^{\beta_{1}}\left(\phi_{p}\left(D_{0^{+}}^{\alpha_{1}} u(t)\right)\right)=f_{1}(t, u(t), v(t)), t \in(0,1), D_{0^{+}}^{\beta_{2}}\left(\phi_{p}\left(D_{0^{+}}^{\alpha_{2}} v(t)\right)\right)=f_{2}(t, u(t), v(t)), t \in(0,1)$, $u(0)=D_{0^{+}}^{q_{1}} u(0)=0, \gamma u(1)+\delta D_{0^{+}}^{q_{2}} u(1)=0, D_{0^{+}}^{\alpha_{1}} u(0)=D_{0^{+}}^{\alpha_{1}} u(1)=0, v(0)=D_{0^{+}}^{q_{1}} v(0)=0, \gamma v(1)+\delta D_{0^{+}}^{q_{2}} v(1)=0, D_{0^{+}}^{\alpha_{2}} v(0)=$ $D_{0^{+}}^{\alpha_{2}} v(1)=0$, by applying five functionals fixed point theorem.

\section{Introduction}

The theory of differential equations offers a broad mathematical basis to understand the problems of modern society which are complex and interdisciplinary by nature. Fractional order differential equations have gained importance due to their applications to almost all areas of science, engineering, and technology. Among all the theories, the most applicable operator is the classical $p$-Laplacian, given by $\phi_{p}(s)=$ $\left.|s|\right|^{p-2} s, p>1$. These types of problems have a wide range of applications in physics and related sciences such as biophysics, plasma physics, and chemical reaction design.

The positive solutions of boundary value problems associated with ordinary differential equations were studied by many authors [1-3] and extended to $p$-Laplacian boundary value problems [4-6]. Later, these results are further extended to fractional order boundary value problems [7-15] by applying various fixed point theorems on cones. Recently, researchers are concentrating on the theory of fractional order boundary value problems associated with $p$-Laplacian operator.

In 2012, Chai [16] investigated the existence and multiplicity of positive solutions for a class of boundary value problem of fractional differential equation with $p$-Laplacian operator,

$$
\begin{aligned}
& D_{0^{+}}^{\beta}\left(\phi_{p}\left(D_{0^{+}}^{\alpha} u(t)\right)\right)+f(t, u(t))=0, \quad 0<t<1, \\
& u(0)=0, \quad u(1)+\sigma D_{0^{+}}^{\gamma} u(1)=0, \quad D_{0^{+}}^{\alpha} u(0)=0,
\end{aligned}
$$

by means of the fixed point theorem on cones.

This paper is concerned with the existence of positive solutions for a coupled system of $p$-Laplacian fractional order boundary value problems:

$$
\begin{gathered}
D_{0^{+}}^{\beta_{1}}\left(\phi_{p}\left(D_{0^{+}}^{\alpha_{1}} u(t)\right)\right)=f_{1}(t, u(t), v(t)), \quad t \in(0,1), \\
D_{0^{+}}^{\beta_{2}}\left(\phi_{p}\left(D_{0^{+}}^{\alpha_{2}} v(t)\right)\right)=f_{2}(t, u(t), v(t)), \quad t \in(0,1), \\
u(0)=D_{0^{+}}^{q_{1}} u(0)=0, \quad \gamma u(1)+\delta D_{0^{+}}^{q_{2}} u(1)=0, \\
D_{0^{+}}^{\alpha_{1}} u(0)=D_{0^{+}}^{\alpha_{1}} u(1)=0, \\
v(0)=D_{0^{+}}^{q_{1}} v(0)=0, \quad \gamma v(1)+\delta D_{0^{+}}^{q_{2}} v(1)=0, \\
D_{0^{+}}^{\alpha_{2}} v(0)=D_{0^{+}}^{\alpha_{2}} v(1)=0,
\end{gathered}
$$


where $\phi_{p}(s)=|s|^{p-2} s, p>1, \phi_{p}^{-1}=\phi_{q}, 1 / p+1 / q=1, \gamma, \delta$ are positive real numbers, $2<\alpha_{i} \leq 3,1<\beta_{i}, q_{i} \leq 2, f_{i}:[0,1] \times$ $\mathbb{R}^{2} \rightarrow \mathbb{R}^{+}$are continuous functions, and $D_{0^{+}}^{\alpha_{i}}, D_{0^{+}}^{\beta_{i}}, D_{0^{+}}^{q_{i}}$, for $i=1,2$ are the standard Riemann-Liouville fractional order derivatives.

The rest of the paper is organized as follows. In Section 2, the Green functions for the homogeneous BVPs corresponding to (2), (4) are constructed and the bounds for the Green functions are estimated. In Section 3, sufficient conditions for the existence of at least three positive solutions for a coupled system of $p$-Laplacian fractional order BVP (2)-(5) are established, by using five functionals fixed point theorem. In Section 4, as an application, the results are demonstrated with an example.

\section{Green Functions and Bounds}

In this section, the Green functions for the homogeneous BVPs are constructed and the bounds for the Green functions are estimated, which are essential to establish the main results. BVP:

Let $G_{1}(t, s)$ be Green's function for the homogeneous

$$
\begin{gathered}
-D_{0^{+}}^{\alpha_{1}} u(t)=0, \quad t \in(0,1), \\
u(0)=0, \quad D_{0^{+}}^{q_{1}} u(0)=0, \quad \gamma u(1)+\delta D_{0^{+}}^{q_{2}} u(1)=0 .
\end{gathered}
$$

Lemma 1. Let $d=\delta \Gamma\left(\alpha_{1}\right)+\gamma \Gamma\left(\alpha_{1}-q_{2}\right) \neq 0$. If $h \in C[0,1]$, then the fractional order $B V P$

$$
D_{0^{+}}^{\alpha_{1}} u(t)+h(t)=0, \quad t \in(0,1),
$$

with (7) has a unique solution

$$
u(t)=\int_{0}^{1} G_{1}(t, s) h(s) d s,
$$

where

$$
\begin{aligned}
G_{1}(t, s) & = \begin{cases}G_{11}(t, s), & 0 \leq t \leq s \leq 1, \\
G_{12}(t, s), & 0 \leq s \leq t \leq 1,\end{cases} \\
G_{11}(t, s) & =\frac{1}{d}\left[\frac{\gamma \Gamma\left(\alpha_{1}-q_{2}\right)}{\Gamma\left(\alpha_{1}\right)}+\delta(1-s)^{-q_{2}}\right][t(1-s)]^{\alpha_{1}-1}, \\
G_{12}(t, s) & =G_{11}(t, s)-\frac{1}{d}\left[\delta+\frac{\gamma \Gamma\left(\alpha_{1}-q_{2}\right)}{\Gamma\left(\alpha_{1}\right)}\right](t-s)^{\alpha_{1}-1} .
\end{aligned}
$$

Proof. Let $u \in C^{\left[\alpha_{1}\right]+1}[0,1]$ be the solution of fractional order $\operatorname{BVP}(8),(7)$. Then

$$
I_{0^{+}}^{\alpha_{1}} D_{0^{+}}^{\alpha_{1}} u(t)=-I_{0^{+}}^{\alpha_{1}} h(t),
$$

and hence

$$
\begin{aligned}
u(t)= & \frac{-1}{\Gamma\left(\alpha_{1}\right)} \int_{0}^{t}(t-s)^{\alpha_{1}-1} h(s) d s \\
& +c_{1} t^{\alpha_{1}-1}+c_{2} t^{\alpha_{1}-2}+c_{3} t^{\alpha_{1}-3} .
\end{aligned}
$$

Using the boundary conditions (7), $c_{1}, c_{2}$, and $c_{3}$ are determined as

$$
\begin{gathered}
c_{1}=\frac{1}{d} \int_{0}^{1}\left[\frac{\gamma \Gamma\left(\alpha_{1}-q_{2}\right)}{\Gamma\left(\alpha_{1}\right)}+\delta(1-s)^{-q_{2}}\right](1-s)^{\alpha_{1}-1} h(s) d s, \\
c_{2}=0, \quad c_{3}=0 .
\end{gathered}
$$

Hence, the unique solution of (8), (7) is

$$
\begin{aligned}
u(t)= & \int_{0}^{t}\left\{\frac{1}{d}\left[\frac{\gamma \Gamma\left(\alpha_{1}-q_{2}\right)}{\Gamma\left(\alpha_{1}\right)}+\delta(1-s)^{-q_{2}}\right][t(1-s)]^{\alpha_{1}-1}\right. \\
& \left.\quad-\frac{(t-s)^{\alpha_{1}-1}}{\Gamma\left(\alpha_{1}\right)}\right\} h(s) d s \\
& +\int_{t}^{1} \frac{1}{d}\left[\frac{\gamma \Gamma\left(\alpha_{1}-q_{2}\right)}{\Gamma\left(\alpha_{1}\right)}+\delta(1-s)^{-q_{2}}\right] \\
& \times[t(1-s)]^{\alpha_{1}-1} h(s) d s \\
= & \int_{0}^{1} G_{1}(t, s) h(s) d s .
\end{aligned}
$$

Lemma 2. Let $y(t) \in C[0,1]$ and $2<\alpha_{1} \leq 3,1<\beta_{1} \leq 2$. Then the fractional order BVP

$$
D_{0^{+}}^{\beta_{1}}\left(\phi_{p}\left(D_{0^{+}}^{\alpha_{1}} u(t)\right)\right)=y(t), \quad t \in(0,1),
$$

with (4) has a unique solution

$$
u(t)=\int_{0}^{1} G_{1}(t, s) \phi_{q}\left(\int_{0}^{1} H_{1}(s, \tau) y(\tau) d \tau\right) d s,
$$

where

$$
H_{1}(t, s)= \begin{cases}\frac{[t(1-s)]^{\beta_{1}-1}}{\Gamma\left(\beta_{1}\right)}, & 0 \leq t \leq s \leq 1, \\ \frac{[t(1-s)]^{\beta_{1}-1}-(t-s)^{\beta_{1}-1}}{\Gamma\left(\beta_{1}\right)}, & 0 \leq s \leq t \leq 1 .\end{cases}
$$

Proof. An equivalent integral equation for (16) is given by

$$
\begin{aligned}
\phi_{p}\left(D_{0^{+}}^{\alpha_{1}} u(t)\right)= & \frac{1}{\Gamma\left(\beta_{1}\right)} \int_{0}^{t}(t-\tau)^{\beta_{1}-1} y(\tau) d \tau \\
& +c_{1} t^{\beta_{1}-1}+c_{2} t^{\beta_{1}-2} .
\end{aligned}
$$

Using the conditions $D_{0^{+}}^{\alpha_{1}} u(0)=0, D_{0^{+}}^{\alpha_{1}} u(1)=0, c_{1}$, and $c_{2}$ are determined as $c_{1}=\left(-1 / \Gamma\left(\beta_{1}\right)\right) \int_{0}^{1}(1-\tau)^{\beta_{1}-1} y(\tau) d \tau$ and $c_{2}=0$. Then,

$$
\begin{aligned}
\phi_{p}\left(D_{0^{+}}^{\alpha_{1}} u(t)\right)= & \frac{1}{\Gamma\left(\beta_{1}\right)} \int_{0}^{t}(t-\tau)^{\beta_{1}-1} y(\tau) d \tau \\
& -\frac{1}{\Gamma\left(\beta_{1}\right)} \int_{0}^{1}[t(1-\tau)]^{\beta_{1}-1} y(\tau) d \tau \\
= & -\int_{0}^{1} H_{1}(t, \tau) y(\tau) d \tau .
\end{aligned}
$$


Therefore,

$$
\phi_{p}^{-1}\left(\phi_{p}\left(D_{0^{+}}^{\alpha_{1}} u(t)\right)\right)=-\phi_{p}^{-1}\left(\int_{0}^{1} H_{1}(t, \tau) y(\tau) d \tau\right) .
$$

Consequently,

$$
D_{0^{+}}^{\alpha_{1}} u(t)+\phi_{q}\left(\int_{0}^{1} H_{1}(t, \tau) y(\tau) d \tau\right)=0
$$

Hence, $u(t)=\int_{0}^{1} G_{1}(t, s) \phi_{q}\left(\int_{0}^{1} H_{1}(s, \tau) y(\tau) d \tau\right) d s$ is the solution of fractional order BVP (16) and (4).

Lemma 3. Assume that $\delta\left(q_{2}-1\right)>\gamma \Gamma\left(\alpha_{1}-q_{2}\right) / \Gamma\left(\alpha_{1}\right)$. Then Green's function $G_{1}(t, s)$ satisfies the following inequalities:

(i) $G_{1}(t, s) \geq 0$, for all $(t, s) \in[0,1] \times[0,1]$,

(ii) $G_{1}(t, s) \leq G_{1}(1, s)$, for all $(t, s) \in[0,1] \times[0,1]$,

(iii) $G_{1}(t, s) \geq\left(1 / 4^{\alpha_{1}-1}\right) G_{1}(1, s)$, for all $(t, s) \in I \times[0,1]$,

where $I=[1 / 4,3 / 4]$.

Proof. Green's function $G_{1}(t, s)$ is given in (10). For $0 \leq t \leq$ $s \leq 1$,

$$
\begin{aligned}
G_{11}(t, s) & =\frac{1}{d}\left[\frac{\gamma \Gamma\left(\alpha_{1}-q_{2}\right)}{\Gamma\left(\alpha_{1}\right)}+\delta(1-s)^{-q_{2}}\right][t(1-s)]^{\alpha_{1}-1} \\
& \geq 0
\end{aligned}
$$

For $0 \leq s \leq t \leq 1$,

$$
\begin{aligned}
G_{12}(t, s)= & {\left[\frac{\gamma \Gamma\left(\alpha_{1}-q_{2}\right)}{\Gamma\left(\alpha_{1}\right)}+\delta(1-s)^{-q_{2}}\right] \frac{[t-t s]^{\alpha_{1}-1}}{d} } \\
& -\left[\delta+\frac{\gamma \Gamma\left(\alpha_{1}-q_{2}\right)}{\Gamma\left(\alpha_{1}\right)}\right] \frac{(t-s)^{\alpha_{1}-1}}{d} \\
\geq & {\left[\frac{\gamma \Gamma\left(\alpha_{1}-q_{2}\right)}{\Gamma\left(\alpha_{1}\right)}+\delta(1-s)^{-q_{2}}\right] \frac{[t-t s]^{\alpha_{1}-1}}{d} } \\
& -\left[\delta+\frac{\gamma \Gamma\left(\alpha_{1}-q_{2}\right)}{\Gamma\left(\alpha_{1}\right)}\right] \frac{(t-t s)^{\alpha_{1}-1}}{d} \\
\geq & \frac{\delta}{d}\left[(1-s)^{-q_{2}}-1\right][t-t s]^{\alpha_{1}-1} \geq 0 .
\end{aligned}
$$

Hence, the inequality (i) is proved. For $0 \leq t \leq s \leq 1$,

$$
\begin{aligned}
\frac{\partial G_{11}(t, s)}{\partial t}= & \frac{1}{d}\left[\frac{\gamma \Gamma\left(\alpha_{1}-q_{2}\right)}{\Gamma\left(\alpha_{1}\right)}+\delta(1-s)^{-q_{2}}\right](1-s)^{\alpha_{1}-1} \\
& \times\left(\alpha_{1}-1\right) t^{\alpha_{1}-2} \geq 0 .
\end{aligned}
$$

Therefore, $G_{11}(t, s)$ is increasing with respect to $t$, which implies that $G_{11}(t, s) \leq G_{11}(1, s)$. Now, for $0 \leq s \leq t \leq 1$,

$$
\frac{\partial G_{12}(t, s)}{\partial t}=\left[\frac{\gamma \Gamma\left(\alpha_{1}-q_{2}\right)}{\Gamma\left(\alpha_{1}\right)}+\delta(1-s)^{-q_{2}}\right]
$$

$$
\begin{aligned}
& \times \frac{\left(\alpha_{1}-1\right)[t-t s]^{\alpha_{1}-2}(1-s)}{d} \\
& -\left[\delta+\frac{\gamma \Gamma\left(\alpha_{1}-q_{2}\right)}{\Gamma\left(\alpha_{1}\right)}\right] \frac{\left(\alpha_{1}-1\right)(t-s)^{\alpha_{1}-2}}{d} \\
& \geq\left[\frac{\gamma \Gamma\left(\alpha_{1}-q_{2}\right)}{\Gamma\left(\alpha_{1}\right)}+\delta(1-s)^{-q_{2}}\right] \\
& \times \frac{\left(\alpha_{1}-1\right)[t-t s]^{\alpha_{1}-2}(1-s)}{d} \\
& -\left[\delta+\frac{\gamma \Gamma\left(\alpha_{1}-q_{2}\right)}{\Gamma\left(\alpha_{1}\right)}\right] \frac{\left(\alpha_{1}-1\right)(t-t s)^{\alpha_{1}-2}}{d}
\end{aligned}
$$

$$
\begin{aligned}
= & \frac{\left(\alpha_{1}-1\right)(t-t s)^{\alpha_{1}-2}}{d} \\
& \times\left[\left(\frac{\gamma \Gamma\left(\alpha_{1}-q_{2}\right)}{\Gamma\left(\alpha_{1}\right)}+\delta(1-s)^{-q_{2}}\right)(1-s)\right.
\end{aligned}
$$$$
\left.-\left(\delta+\frac{\gamma \Gamma\left(\alpha_{1}-q_{2}\right)}{\Gamma\left(\alpha_{1}\right)}\right)\right]
$$

$=\frac{\left(\alpha_{1}-1\right)(t-t s)^{\alpha_{1}-2}}{d}$

$$
\begin{gathered}
\times\left[\delta\left((1-s)^{-\left(q_{2}-1\right)}-1\right)-\frac{\gamma \Gamma\left(\alpha_{1}-q_{2}\right)}{\Gamma\left(\alpha_{1}\right)} s\right] \\
=\frac{\left(\alpha_{1}-1\right)(t-t s)^{\alpha_{1}-2}}{d} \\
\times\left[\delta\left(\left(q_{2}-1\right) s+\frac{\left(q_{2}-1\right) q_{2}}{2} s^{2}+\cdots\right)\right. \\
\left.\quad-\frac{\gamma \Gamma\left(\alpha_{1}-q_{2}\right)}{\Gamma\left(\alpha_{1}\right)} s\right]
\end{gathered}
$$

$$
\begin{aligned}
= & \frac{\left(\alpha_{1}-1\right)(t-t s)^{\alpha_{1}-2}}{d} \\
& \times\left[\left(\delta\left(q_{2}-1\right)-\frac{\gamma \Gamma\left(\alpha_{1}-q_{2}\right)}{\Gamma\left(\alpha_{1}\right)}\right) s+O\left(s^{2}\right)\right]
\end{aligned}
$$
$\geq 0$. 
Therefore, $G_{12}(t, s)$ is increasing with respect to $t$, which implies that $G_{12}(t, s) \leq G_{12}(1, s)$. Hence, the inequality (ii) is proved. Now, the inequality (iii) can be established.

Let $0 \leq t \leq s \leq 1$ and $t \in I$. Then

$$
\begin{aligned}
G_{11}(t, s) & =\left[\frac{\gamma \Gamma\left(\alpha_{1}-q_{2}\right)}{\Gamma\left(\alpha_{1}\right)}+\delta(1-s)^{-q_{2}}\right][t(1-s)]^{\alpha_{1}-1} \\
& =t^{\alpha_{1}-1}\left[\frac{\gamma \Gamma\left(\alpha_{1}-q_{2}\right)}{\Gamma\left(\alpha_{1}\right)}+\delta(1-s)^{-q_{2}}\right](1-s)^{\alpha_{1}-1} \\
& =t^{\alpha_{1}-1} G_{11}(1, s) \\
& \geq \frac{1}{4^{\alpha_{1}-1}} G_{11}(1, s) .
\end{aligned}
$$

Let $0 \leq s \leq t \leq 1$ and $t \in I$. Then

$$
\begin{aligned}
& G_{12}(t, s)= {\left[\frac{\gamma \Gamma\left(\alpha_{1}-q_{2}\right)}{\Gamma\left(\alpha_{1}\right)}+\delta(1-s)^{-q_{2}}\right][t(1-s)]^{\alpha_{1}-1} } \\
&-\left[\delta+\frac{\gamma \Gamma\left(\alpha_{1}-q_{2}\right)}{\Gamma\left(\alpha_{1}\right)}\right](t-s)^{\alpha_{1}-1} \\
& \geq {\left[\frac{\gamma \Gamma\left(\alpha_{1}-q_{2}\right)}{\Gamma\left(\alpha_{1}\right)}+\delta(1-s)^{-q_{2}}\right][t(1-s)]^{\alpha_{1}-1} } \\
&-\left[\delta+\frac{\gamma \Gamma\left(\alpha_{1}-q_{2}\right)}{\Gamma\left(\alpha_{1}\right)}\right](t-t s)^{\alpha_{1}-1} \\
&= t^{\alpha_{1}-1}\left[\frac{\gamma \Gamma\left(\alpha_{1}-q_{2}\right)}{\Gamma\left(\alpha_{1}\right)}+\delta(1-s)^{-q_{2}}\right. \\
& \geq \frac{1}{4^{\alpha_{1}-1}} G_{12}(1, s) . \\
&= t^{\alpha_{1}-1} G_{12}(1, s) \\
&\left.-\left(\delta+\frac{\gamma \Gamma\left(\alpha_{1}-q_{2}\right)}{\Gamma\left(\alpha_{1}\right)}\right)\right](1-s)^{\alpha_{1}-1}
\end{aligned}
$$

Hence the inequality (iii) is proved.

Lemma 4. For $t, s \in[0,1]$, Green's function $H_{1}(t, s)$ satisfies the following inequalities:

(i) $H_{1}(t, s) \geq 0$,

(ii) $H_{1}(t, s) \leq H_{1}(s, s)$.

Proof. Green's function $H_{1}(t, s)$ is given in (18). Clearly, it is observed that, for $0 \leq t \leq s \leq 1, H_{1}(t, s) \geq 0$.

$$
\text { For } 0 \leq s \leq t \leq 1 \text {, }
$$

$$
\begin{aligned}
H_{1}(t, s) & =\frac{1}{\Gamma\left(\beta_{1}\right)}\left[t^{\beta_{1}-1}(1-s)^{\beta_{1}-1}-(t-s)^{\beta_{1}-1}\right] \\
& \geq \frac{1}{\Gamma\left(\beta_{1}\right)}\left[t^{\beta_{1}-1}(1-s)^{\beta_{1}-1}-(t-t s)^{\beta_{1}-1}\right] \\
& =\frac{1}{\Gamma\left(\beta_{1}\right)}\left[t^{\beta_{1}-1}(1-s)^{\beta_{1}-1}-t^{\beta_{1}-1}(1-s)^{\beta_{1}-1}\right] \\
& =0 .
\end{aligned}
$$

Hence, the inequality (i) is proved. Now we establish the inequality (ii), for $0 \leq t \leq s \leq 1$,

$$
\frac{\partial H_{1}(t, s)}{\partial t}=\frac{1}{\Gamma\left(\beta_{1}\right)}\left[\left(\beta_{1}-1\right) t^{\beta_{1}-2}(1-s)^{\beta_{1}-1}\right]>0 .
$$

Therefore, $H_{1}(t, s)$ is increasing with respect to $t$, for $s \in[0,1)$, which implies that $H_{1}(t, s) \leq H_{1}(s, s)$. Similarly, it can be proved that $H_{1}(t, s) \leq H_{1}(s, s)$ for $0 \leq s \leq t \leq 1$. Hence the inequality (ii) is proved.

Lemma 5. Green's function $H_{1}(t, s)$ satisfies the following inequality: $(A)$ there exists a positive function $\gamma_{1}^{*}(s) \in C(0,1)$ such that

$$
\min _{t \in I} H_{1}(t, s) \geq \gamma_{1}^{*}(s) H_{1}(s, s), \quad \text { for } 0<s<1
$$

Proof. Since $H_{1}(t, s)$ is monotonic function, for all $t, s \in$ $[0,1]$, we have

$$
\max _{0 \leq t \leq 1} H_{1}(t, s)=H_{1}(s, s)=\frac{1}{\Gamma\left(\beta_{1}\right)}[s(1-s)]^{\beta_{1}-1}
$$

From (i) of Lemma $4, H_{1}(t, s) \geq 0$, for $t, s \in[0,1]$. For $s \in(0,1 / 4), H_{1}(t, s)$ is increasing with respect to $t$ for $t \in\left(0, s /\left(1-(1-s)^{\left(\beta_{1}-1\right) /\left(\beta_{1}-2\right)}\right)\right)$ and decreasing with respect to $t$ for $t \in\left(s /\left(1-(1-s)^{\left(\beta_{1}-1\right) /\left(\beta_{1}-2\right)}\right), 1 / 4\right)$. For $s \in$ $(1 / 4,1), H_{1}(t, s)$ is decreasing with respect to $t$ for $s \leq t$ and increasing with respect to $t$ for $s \geq t$. If we define

$$
\begin{gathered}
h_{1}(t, s)=\frac{[t(1-s)]^{\beta_{1}-1}-(t-s)^{\beta_{1}-1}}{\Gamma\left(\beta_{1}\right)}, \\
h_{2}(t, s)=\frac{[t(1-s)]^{\beta_{1}-1}}{\Gamma\left(\beta_{1}\right)} .
\end{gathered}
$$


Then,

$$
\begin{aligned}
& \min _{t \in I} H_{1}(t, s)= \begin{cases}h_{1}\left(\frac{3}{4}, s\right), & s \in\left(0, \frac{1}{4}\right], \\
\min \left\{h_{1}\left(\frac{3}{4}, s\right), h_{2}\left(\frac{1}{4}, s\right)\right\}, & s \in\left[\frac{1}{4}, \frac{3}{4}\right], \\
h_{2}\left(\frac{1}{4}, s\right), & s \in\left[\frac{3}{4}, 1\right),\end{cases} \\
& = \begin{cases}h_{1}\left(\frac{3}{4}, s\right), & s \in(0, \xi], \\
h_{2}\left(\frac{1}{4}, s\right), & s \in[\xi, 1),\end{cases} \\
& \left\{\begin{array}{l}
\frac{[(3 / 4)(1-s)]^{\beta_{1}-1}-((3 / 4)-s)^{\beta_{1}-1}}{\Gamma\left(\beta_{1}\right)}, \\
s \in(0, \xi], \\
\frac{1}{\Gamma\left(\beta_{1}\right)} \frac{(1-s)^{\beta_{1}-1}}{4^{\beta_{1}-1}}, \\
s \in[\xi, 1),
\end{array}\right. \\
& \geq\left\{\begin{array}{l}
\frac{[(3 / 4)(1-s)]^{\beta_{1}-1}-((3 / 4)-s)^{\beta_{1}-1}}{[s(1-s)]^{\beta_{1}-1}} \\
\times H_{1}(s, s), \quad s \in(0, \xi] \\
\frac{1}{(4 s)^{\beta_{1}-1}} H_{1}(s, s) \\
s \in[\xi, 1),
\end{array}\right. \\
& =\gamma_{1}^{*}(s) H_{1}(s, s),
\end{aligned}
$$

where

$$
\gamma_{1}^{*}(s)= \begin{cases}\frac{[(3 / 4)(1-s)]^{\beta_{1}-1}-((3 / 4)-s)^{\beta_{1}-1}}{[s(1-s)]^{\beta_{1}-1}}, & s \in(0, \xi], \\ \frac{1}{(4 s)^{\beta_{1}-1}}, & s \in[\xi, 1),\end{cases}
$$

and $\xi \in(1 / 4,3 / 4)$ satisfy the equation $[(3 / 4)(1-\xi)]^{\beta_{1}-1}-$ $((3 / 4)-\xi)^{\beta_{1}-1}=((1-\xi) / 4)^{\beta_{1}-1}$. In particular, $\xi=0.5$ if $\beta_{1}=$ $2 ; \xi \rightarrow 0.5$ as $\beta_{1} \rightarrow 2$; and $\xi \rightarrow 0.76$ as $\beta_{1} \rightarrow 1$. Hence the inequality in (31) holds.

In a similar manner, the results of the Green functions $G_{2}(t, s)$ and $H_{2}(t, s)$ for the homogeneous BVPs corresponding to the fractional order BVP (3) and (5) are obtained.

Remark 6. Consider the following.

$G_{1}(t, s) \geq \eta G_{1}(1, s)$ and $G_{2}(t, s) \geq \eta G_{2}(1, s)$, for all $(t, s) \in$ $I \times[0,1]$, where $\eta=\min \left\{1 / 4^{\alpha_{1}-1}, 1 / 4^{\alpha_{2}-1}\right\}$.

Remark 7. Consider the following.

$H_{1}(t, s) \geq \gamma^{*}(s) H_{1}(s, s)$ and $H_{2}(t, s) \geq \gamma^{*}(s) H_{2}(s, s)$, for all $(t, s) \in I \times[0,1]$, where $\gamma^{*}(s)=\min \left\{\gamma_{1}^{*}(s), \gamma_{2}^{*}(s)\right\}$.

\section{Existence of Multiple Positive Solutions}

In this section, the existence of at least three positive solutions for a coupled system of $p$-Laplacian fractional order BVP (2)-(5) is established by using five functionals fixed point theorem.

Let $\gamma, \beta, \theta$ be nonnegative continuous convex functionals on $P$ and let $\alpha, \psi$ be nonnegative continuous concave functionals on $P$; then for nonnegative numbers $h^{\prime}, a^{\prime}, b^{\prime}, d^{\prime}$, and $c^{\prime}$, convex sets are defined:

$$
\begin{gathered}
P\left(\gamma, c^{\prime}\right)=\left\{y \in P: \gamma(y)<c^{\prime}\right\}, \\
P\left(\gamma, \alpha, a^{\prime}, c^{\prime}\right)=\left\{y \in P: a^{\prime} \leq \alpha(y) ; \gamma(y) \leq c^{\prime}\right\}, \\
Q\left(\gamma, \beta, d^{\prime}, c^{\prime}\right)=\left\{y \in P: \beta(y) \leq d^{\prime} ; \gamma(y) \leq c^{\prime}\right\}, \\
P\left(\gamma, \theta, \alpha, a^{\prime}, b^{\prime}, c^{\prime}\right)=\left\{y \in P: a^{\prime} \leq \alpha(y) ; \theta(y)\right. \\
\left.\leq b^{\prime} ; \gamma(y) \leq c^{\prime}\right\}, \\
Q\left(\gamma, \beta, \psi, h^{\prime}, d^{\prime}, c^{\prime}\right)=\left\{y \in P: h^{\prime} \leq \psi(y) ; \beta(y)\right. \\
\left.\leq d^{\prime} ; \gamma(y) \leq c^{\prime}\right\} .
\end{gathered}
$$

In obtaining multiple positive solutions of the p-Laplacian fractional order BVP (2)-(5), the following so-called five functionals fixed point theorem is fundamental.

Theorem 8 (see [17]). Let $P$ be a cone in the real Banach space $B$. Suppose that $\alpha$ and $\psi$ are nonnegative continuous concave functionals on $P$ and $\gamma, \beta, \theta$ are nonnegative continuous convex functionals on $P$, such that, for some positive numbers $c^{\prime}$ and $e^{\prime}, \alpha(y) \leq \beta(y)$ and $\|y\| \leq e^{\prime} \gamma(y)$, for all $y \in \overline{P\left(\gamma, c^{\prime}\right)}$. Suppose further that $T: \overline{P\left(\gamma, c^{\prime}\right)} \rightarrow \overline{P\left(\gamma, c^{\prime}\right)}$ is completely continuous and there exist constants $h^{\prime}, d^{\prime}, a^{\prime}$, and $b^{\prime} \geq 0$ with $0<d^{\prime}<a^{\prime}$ such that each of the following is satisfied:

(B1) $\left\{y \in P\left(\gamma, \theta, \alpha, a^{\prime}, b^{\prime}, c^{\prime}\right): \alpha(y)>a^{\prime}\right\} \neq \emptyset$ and $\alpha(T y)>$ $a^{\prime}$ for $y \in P\left(\gamma, \theta, \alpha, a^{\prime}, b^{\prime}, c^{\prime}\right)$,

(B2) $\left\{y \in Q\left(\gamma, \beta, \psi, h^{\prime}, d^{\prime}, c^{\prime}\right): \beta(y)>d^{\prime}\right\} \neq \emptyset$ and $\beta(T y)>$ $d^{\prime}$ for $y \in Q\left(\gamma, \beta, \psi, h^{\prime}, d^{\prime}, c^{\prime}\right)$,

(B3) $\alpha(T y)>a^{\prime}$ provided that $y \in P\left(\gamma, \alpha, a^{\prime}, c^{\prime}\right)$ with $\theta(T y)>b^{\prime}$

(B4) $\beta(T y)<d^{\prime}$ provided that $y \in Q\left(\gamma, \beta, \psi, h^{\prime}, d^{\prime}, c^{\prime}\right)$ with $\psi(T y)<h^{\prime}$.

Then, $T$ has at least three fixed points $y_{1}, y_{2}, y_{3} \in \overline{P\left(\gamma, c^{\prime}\right)}$ such that $\beta\left(y_{1}\right)<d^{\prime}, a^{\prime}<\alpha\left(y_{2}\right)$ and $d^{\prime}<\beta\left(y_{3}\right)$ with $\alpha\left(y_{3}\right)<a^{\prime}$.

Consider the Banach space $B=E \times E$, where $E=\{u: u \in$ $C[0,1]\}$ equipped with the norm $\|(u, v)\|=\|u\|_{0}+\|v\|_{0}$, for $(u, v) \in B$ and the norm, is defined as

$$
\|u\|_{0}=\max _{0 \leq t \leq 1}|u(t)|
$$


Define a cone $P \subset B$ by

$$
\begin{gathered}
P=\{(u, v) \in B \mid u(t) \geq 0, v(t) \geq 0, t \in[0,1], \\
\left.\min _{t \in I}[u(t)+v(t)] \geq \eta\|(u, v)\|\right\} .
\end{gathered}
$$

Define the nonnegative continuous concave functionals $\alpha, \psi$ and the nonnegative continuous convex functionals $\beta, \theta, \gamma$ on $P$ by

$$
\begin{aligned}
& \alpha(u, v)=\min _{t \in I}\{|u|+|v|\}, \\
& \psi(u, v)=\min _{t \in I_{1}}\{|u|+|v|\}, \\
& \gamma(u, v)=\max _{t \in[0,1]}\{|u|+|v|\}, \\
& \beta(u, v)=\max _{t \in I_{1}}\{|u|+|v|\}, \\
& \theta(u, v)=\max _{t \in I}\{|u|+|v|\},
\end{aligned}
$$

where $I_{1}=[1 / 3,2 / 3]$. For any $(u, v) \in P$,

$$
\begin{gathered}
\alpha(u, v)=\min _{t \in I}\{|u|+|v|\} \leq \max _{t \in I_{1}}\{|u|+|v|\}=\beta(u, v), \\
\|(u, v)\| \leq \frac{1}{\eta} \min _{t \in I}\{|u|+|v|\} \leq \frac{1}{\eta} \max _{t \in[0,1]}\{|u|+|v|\}=\frac{1}{\eta} \gamma(u, v) .
\end{gathered}
$$

Let

$$
\begin{aligned}
& L=\min \{\left(\int_{0}^{1} G_{1}(1, s) \phi_{q}\left(\int_{0}^{1} H_{1}(\tau, \tau) d \tau\right) d s\right)^{-1}, \\
&\left.\left(\int_{0}^{1} G_{2}(1, s) \phi_{q}\left(\int_{0}^{1} H_{2}(\tau, \tau) d \tau\right) d s\right)^{-1}\right\}, \\
& M=\max \{\left(\int_{s \in I} \eta G_{1}(1, s) \phi_{q}\left(\int_{\tau \in I} \gamma^{*}(\tau) H_{1}(\tau, \tau) d \tau\right) d s\right)^{-1}, \\
&\left(\int_{s \in I} \eta G_{2}(1, s) \phi_{q}\right. \\
&\left.\left.\times\left(\int_{\tau \in I} \gamma^{*}(\tau) H_{2}(\tau, \tau) d \tau\right) d s\right)^{-1}\right\} .
\end{aligned}
$$

Theorem 9. Suppose that there exist $0<a^{\prime}<b^{\prime}<b^{\prime} / \eta<c^{\prime}$ such that $f_{i}$, for $i=1,2$ satisfies the following conditions:

(A1) $f_{i}(t, u(t), v(t))<\phi_{p}\left(a^{\prime} L / 2\right), t \in[0,1]$ and $u, v \in$ $\left[\eta a^{\prime}, a^{\prime}\right]$

(A2) $f_{i}(t, u(t), v(t))>\phi_{p}\left(b^{\prime} M / 2\right), t \in I$ and $u, v \in$ $\left[b^{\prime}, b^{\prime} / \eta\right]$,

(A3) $f_{i}(t, u(t), v(t))<\phi_{p}\left(c^{\prime} L / 2\right), t \in[0,1]$ and $u, v \in$ $\left[0, c^{\prime}\right]$.
Then, the fractional order BVP (2)-(5) has at least three positive solutions, $\left(x_{1}, x_{2}\right),\left(y_{1}, y_{2}\right)$, and $\left(z_{1}, z_{2}\right)$ such that $\beta\left(x_{1}, x_{2}\right)<$ $a^{\prime}, b^{\prime}<\alpha\left(y_{1}, y_{2}\right)$ and $a^{\prime}<\beta\left(z_{1}, z_{2}\right)$ with $\alpha\left(z_{1}, z_{2}\right)<b^{\prime}$.

Proof. Let $T_{1}, T_{2}: P \rightarrow E$ and $T: P \rightarrow B$ be the operators defined by

$$
\begin{aligned}
& T_{1}(u, v)(t)=\int_{0}^{1} G_{1}(t, s) \phi_{q} \\
& \times\left(\int_{0}^{1} H_{1}(s, \tau) f_{1}(\tau, u(\tau), v(\tau)) d \tau\right) d s \\
& T_{2}(u, v)(t)=\int_{0}^{1} G_{2}(t, s) \phi_{q} \\
& \times\left(\int_{0}^{1} H_{2}(s, \tau) f_{2}(\tau, u(\tau), v(\tau)) d \tau\right) d s \\
& T(u, v)(t)=\left(T_{1}(u, v)(t), T_{2}(u, v)(t)\right), \quad \text { for }(u, v) \in B \text {. }
\end{aligned}
$$

It is obvious that a fixed point of $T$ is the solution of the fractional order BVP (2)-(5). Three fixed points of $T$ are sought. First, it is shown that $T: P \rightarrow P$. Let $(u, v) \in P$. Clearly, $T_{1}(u, v)(t) \geq 0$ and $T_{2}(u, v)(t) \geq 0$, for $t \in[0,1]$. Also, for $(u, v) \in P$,

$$
\begin{aligned}
& \left\|T_{1}(u, v)\right\|_{0} \leq \int_{0}^{1} G_{1}(1, s) \phi_{q} \\
& \times\left(\int_{0}^{1} H_{1}(s, \tau) f_{1}(\tau, u(\tau), v(\tau)) d \tau\right) d s \\
& \left\|T_{2}(u, v)\right\|_{0} \leq \int_{0}^{1} G_{2}(1, s) \phi_{q} \\
& \times\left(\int_{0}^{1} H_{2}(s, \tau) f_{2}(\tau, u(\tau), v(\tau)) d \tau\right) d s \\
& \min _{t \in I} T_{1}(u, v)(t)=\min _{t \in I} \int_{0}^{1} G_{1}(t, s) \phi_{q} \\
& \times\left(\int_{0}^{1} H_{1}(s, \tau) f_{1}(\tau, u(\tau), v(\tau)) d \tau\right) d s \\
& \geq \eta \int_{0}^{1} G_{1}(1, s) \phi_{q} \\
& \times\left(\int_{0}^{1} H_{1}(s, \tau) f_{1}(\tau, u(\tau), v(\tau)) d \tau\right) d s \\
& \geq \eta\left\|T_{1}(u, v)\right\|_{0} .
\end{aligned}
$$


Similarly, $\min _{t \in I} T_{2}(u, v)(t) \geq \eta\left\|T_{2}(u, v)\right\|_{0}$. Therefore,

$$
\begin{aligned}
& \min _{t \in I}\left\{T_{1}(u, v)(t)+T_{2}(u, v)(t)\right\} \\
& \geq \eta\left\|T_{1}(u, v)\right\|_{0}+\eta\left\|T_{2}(u, v)\right\|_{0} \\
& =\eta\left(\left\|T_{1}(u, v)\right\|_{0}+\left\|T_{2}(u, v)\right\|_{0}\right) \\
& =\eta\left\|\left(T_{1}(u, v), T_{2}(u, v)\right)\right\| \\
& =\eta\|T(u, v)\| .
\end{aligned}
$$

Hence, $T(u, v) \in P$ and so $T: P \rightarrow P$. Moreover, $T$ is completely continuous operator. From $(40)$, for each $(u, v) \in$ $P, \alpha(u, v) \leq \beta(u, v)$, and $\|(u, v)\| \leq(1 / \eta) \gamma(u, v)$. It is shown that $T: \overline{P\left(\gamma, c^{\prime}\right)} \rightarrow \overline{P\left(\gamma, c^{\prime}\right)}$. Let $(u, v) \in \overline{P\left(\gamma, c^{\prime}\right)}$. Then $0 \leq|u|+|v| \leq c^{\prime}$. Condition (A3) is used to obtain

$$
\begin{aligned}
& \gamma(T(u, v)(t)) \\
& =\max _{t \in[0,1]}\left[\int_{0}^{1} G_{1}(t, s) \phi_{q}\right. \\
& \quad \times\left(\int_{0}^{1} H_{1}(s, \tau) f_{1}(\tau, u(\tau), v(\tau)) d \tau\right) d s \\
& \quad+\int_{0}^{1} G_{2}(t, s) \phi_{q} \\
& \leq \int_{0}^{1} G_{1}(t, s) \phi_{q}\left(\int_{0}^{1} H_{1}(s, \tau) \phi_{p}\left(\frac{c^{\prime} L}{2}\right) d \tau\right) d s \\
& \left.\left.+\int_{0}^{1} G_{2}^{1} H_{2}(s, \tau) f_{2}(\tau, u(\tau), v(\tau)) d \tau\right) d s\right] \\
& <\frac{c^{\prime} L}{2} \int_{0}^{1} G_{1}(1, s) \phi_{q}\left(\int_{0}^{1} H_{1}(\tau, \tau) d \tau\right) d s \\
& \left.+\frac{c^{\prime} L}{2} \int_{0}^{1} G_{2}(s, \tau) \phi_{p}\left(\frac{c^{\prime} L}{2}\right) d \tau\right) d s \\
& \quad+\frac{c^{\prime}}{2}(1, s) \phi_{q}\left(\int_{0}^{1} H_{2}(\tau, \tau) d \tau\right) d s
\end{aligned}
$$

Therefore $T: \overline{P\left(\gamma, c^{\prime}\right)} \rightarrow \overline{P\left(\gamma, c^{\prime}\right)}$. Now conditions (B1) and (B2) of Theorem 8 are to be verified. It is obvious that

$\frac{b^{\prime}+\left(b^{\prime} / \eta\right)}{2} \in\left\{(u, v) \in P\left(\gamma, \theta, \alpha, b^{\prime}, \frac{b^{\prime}}{\eta}, c^{\prime}\right): \alpha(u, v)>b^{\prime}\right\} \neq \emptyset$, $\frac{\eta a^{\prime}+a^{\prime}}{2} \in\left\{(u, v) \in Q\left(\gamma, \beta, \psi, \eta a^{\prime}, a^{\prime}, c^{\prime}\right): \beta(u, v)<a^{\prime}\right\} \neq \emptyset$.

Next, let $(u, v) \in P\left(\gamma, \theta, \alpha, b^{\prime}, b^{\prime} / \eta, c^{\prime}\right)$ or $(u, v) \quad \epsilon$ $Q\left(\gamma, \beta, \psi, \eta a^{\prime}, a^{\prime}, c^{\prime}\right)$. Then, $b^{\prime} \leq|u(t)|+|v(t)| \leq b^{\prime} / \eta$ and $\eta a^{\prime} \leq|u(t)|+|v(t)| \leq a^{\prime}$. Now, condition (A2) is applied to get

$$
\begin{aligned}
& \alpha(T(u, v)(t)) \\
& =\min _{t \in I}\left[\int_{0}^{1} G_{1}(t, s) \phi_{q}\left(\int_{0}^{1} H_{1}(s, \tau) f_{1}(\tau, u(\tau), v(\tau)) d \tau\right) d s\right. \\
& +\int_{0}^{1} G_{2}(t, s) \phi_{q} \\
& \left.\times\left(\int_{0}^{1} H_{2}(s, \tau) f_{2}(\tau, u(\tau), v(\tau)) d \tau\right) d s\right] \\
& \geq \eta\left[\int_{0}^{1} G_{1}(1, s) \phi_{q}\left(\int_{0}^{1} \gamma^{*}(\tau) H_{1}(\tau, \tau) \phi_{p}\left(\frac{b^{\prime} M}{2}\right) d \tau\right) d s\right. \\
& +\int_{0}^{1} G_{2}(1, s) \phi_{q} \\
& \left.\times\left(\int_{0}^{1} \gamma^{*}(\tau) H_{2}(\tau, \tau) \phi_{p}\left(\frac{b^{\prime} M}{2}\right) d \tau\right) d s\right] \\
& >\frac{b^{\prime} M}{2} \int_{s \in I} \eta G_{1}(1, s) \phi_{q}\left(\int_{\tau \in I} \gamma^{*}(\tau) H_{1}(\tau, \tau) d \tau\right) d s \\
& +\frac{b^{\prime} M}{2} \int_{s \in I} \eta G_{2}(1, s) \phi_{q}\left(\int_{\tau \in I} \gamma^{*}(\tau) H_{2}(\tau, \tau) d \tau\right) d s \\
& \geq \frac{b^{\prime}}{2}+\frac{b^{\prime}}{2}=b^{\prime} \text {. }
\end{aligned}
$$

Clearly, condition (A1) leads to

$$
\begin{aligned}
& \beta(T(u, v)(t)) \\
& =\max _{t \in I_{1}}\left[\int_{0}^{1} G_{1}(t, s) \phi_{q}\left(\int_{0}^{1} H_{1}(s, \tau) f_{1}(\tau, u(\tau), v(\tau)) d \tau\right) d s\right. \\
& \quad+\int_{0}^{1} G_{2}(t, s) \phi_{q} \\
& \left.\quad \times\left(\int_{0}^{1} H_{2}(s, \tau) f_{2}(\tau, u(\tau), v(\tau)) d \tau\right) d s\right] \\
& \leq \int_{0}^{1} G_{1}(1, s) \phi_{q}\left(\int_{0}^{1} H_{1}(s, \tau) \phi_{p}\left(\frac{a^{\prime} L}{2}\right) d \tau\right) d s \\
& \quad+\int_{0}^{1} G_{2}(1, s) \phi_{q}\left(\int_{0}^{1} H_{2}(s, \tau) \phi_{p}\left(\frac{a^{\prime} L}{2}\right) d \tau\right) d s \\
& <\frac{a^{\prime} L}{2} \int_{0}^{1} G_{1}(1, s) \phi_{q}\left(\int_{0}^{1} H_{1}(\tau, \tau) d \tau\right) d s \\
& \quad+\frac{a^{\prime} L}{2} \int_{0}^{1} G_{2}(1, s) \phi_{q}\left(\int_{0}^{1} H_{2}(\tau, \tau) d \tau\right) d s \\
& \leq \frac{a^{\prime}}{2}+\frac{a^{\prime}}{2}=a^{\prime} .
\end{aligned}
$$


To see that (B3) is satisfied, let $(u, v) \in P\left(\gamma, \alpha, b^{\prime}, c^{\prime}\right)$ with $\theta(T(u, v)(t))>b^{\prime} / \eta$. Then

$$
\begin{aligned}
& \alpha(T(u, v)(t)) \\
& =\min _{t \in I}\left[\int_{0}^{1} G_{1}(t, s) \phi_{q}\left(\int_{0}^{1} H_{1}(s, \tau) f_{1}(\tau, u(\tau), v(\tau)) d \tau\right) d s\right. \\
& +\int_{0}^{1} G_{2}(t, s) \phi_{q} \\
& \left.\times\left(\int_{0}^{1} H_{2}(s, \tau) f_{2}(\tau, u(\tau), v(\tau)) d \tau\right) d s\right] \\
& \geq \eta\left[\int_{0}^{1} G_{1}(1, s) \phi_{q}\left(\int_{0}^{1} H_{1}(s, \tau) f_{1}(\tau, u(\tau), v(\tau)) d \tau\right) d s\right. \\
& +\int_{0}^{1} G_{2}(1, s) \phi_{q} \\
& \left.\times\left(\int_{0}^{1} H_{2}(s, \tau) f_{2}(\tau, u(\tau), v(\tau)) d \tau\right) d s\right] \\
& \geq \eta \max _{t \in[0,1]}\left[\int_{0}^{1} G_{1}(t, s) \phi_{q}\right. \\
& \times\left(\int_{0}^{1} H_{1}(s, \tau) f_{1}(\tau, u(\tau), v(\tau)) d \tau\right) d s \\
& +\int_{0}^{1} G_{2}(t, s) \phi_{q} \\
& \left.\times\left(\int_{0}^{1} H_{2}(s, \tau) f_{2}(\tau, u(\tau), v(\tau)) d \tau\right) d s\right] \\
& \geq \eta \max _{t \in I}\left[\int_{0}^{1} G_{1}(t, s) \phi_{q}\right. \\
& \times\left(\int_{0}^{1} H_{1}(s, \tau) f_{1}(\tau, u(\tau), v(\tau)) d \tau\right) d s \\
& +\int_{0}^{1} G_{2}(t, s) \phi_{q} \\
& \left.\times\left(\int_{0}^{1} H_{2}(s, \tau) f_{2}(\tau, u(\tau), v(\tau)) d \tau\right) d s\right]
\end{aligned}
$$$$
=\eta \theta(T(u, v)(t))>b^{\prime} \text {. }
$$

Finally, it is shown that (B4) holds. Let $(u, v) \in Q\left(\gamma, \beta, a^{\prime}, c^{\prime}\right)$ with $\psi(T(u, v))<\eta a^{\prime}$. Then, we have

$\beta(T(u, v)(t))$

$$
\begin{gathered}
=\max _{t \in I_{1}}\left[\int_{0}^{1} G_{1}(t, s) \phi_{q}\left(\int_{0}^{1} H_{1}(s, \tau) f_{1}(\tau, u(\tau), v(\tau)) d \tau\right) d s\right. \\
+\int_{0}^{1} G_{2}(t, s) \phi_{q}
\end{gathered}
$$

$$
\begin{aligned}
& \left.\times\left(\int_{0}^{1} H_{2}(s, \tau) f_{2}(\tau, u(\tau), v(\tau)) d \tau\right) d s\right] \\
& \leq \max _{t \in[0,1]}\left[\int_{0}^{1} G_{1}(t, s) \phi_{q}\left(\int_{0}^{1} H_{1}(s, \tau) f_{1}(\tau, u(\tau), v(\tau)) d \tau\right) d s\right. \\
& +\int_{0}^{1} G_{2}(t, s) \phi_{q} \\
& \left.\times\left(\int_{0}^{1} H_{2}(s, \tau) f_{2}(\tau, u(\tau), v(\tau)) d \tau\right) d s\right] \\
& \leq \int_{0}^{1} G_{1}(1, s) \phi_{q}\left(\int_{0}^{1} H_{1}(s, \tau) f_{1}(\tau, u(\tau), v(\tau)) d \tau\right) d s \\
& +\int_{0}^{1} G_{2}(1, s) \phi_{q}\left(\int_{0}^{1} H_{2}(s, \tau) f_{2}(\tau, u(\tau), v(\tau)) d \tau\right) d s \\
& =\frac{1}{\eta}\left[\eta \int_{0}^{1} G_{1}(1, s) \phi_{q}\left(\int_{0}^{1} H_{1}(s, \tau) f_{1}(\tau, u(\tau), v(\tau)) d \tau\right) d s\right. \\
& +\eta \int_{0}^{1} G_{2}(1, s) \phi_{q} \\
& \left.\times\left(\int_{0}^{1} H_{2}(s, \tau) f_{2}(\tau, u(\tau), v(\tau)) d \tau\right) d s\right] \\
& \leq \frac{1}{\eta} \min _{t \in I}\left[\int_{0}^{1} G_{1}(t, s) \phi_{q}\left(\int_{0}^{1} H_{1}(s, \tau) f_{1}(\tau, u(\tau), v(\tau)) d \tau\right) d s\right. \\
& +\int_{0}^{1} G_{2}(t, s) \phi_{q} \\
& \left.\times\left(\int_{0}^{1} H_{2}(s, \tau) f_{2}(\tau, u(\tau), v(\tau)) d \tau\right) d s\right] \\
& \leq \frac{1}{\eta} \min _{t \in I_{1}}\left[\int_{0}^{1} G_{1}(t, s) \phi_{q}\left(\int_{0}^{1} H_{1}(s, \tau) f_{1}(\tau, u(\tau), v(\tau)) d \tau\right) d s\right. \\
& +\int_{0}^{1} G_{2}(t, s) \phi_{q} \\
& \left.\times\left(\int_{0}^{1} H_{2}(s, \tau) f_{2}(\tau, u(\tau), v(\tau)) d \tau\right) d s\right] \\
& =\frac{1}{\eta} \psi(T(u, v)(t))<a^{\prime}
\end{aligned}
$$

It has been proved that all the conditions of Theorem 8 are satisfied. Therefore, the fractional order BVP (2)-(5) has at least three positive solutions, $\left(x_{1}, x_{2}\right),\left(y_{1}, y_{2}\right)$, and $\left(z_{1}, z_{2}\right)$ such that $\beta\left(x_{1}, x_{2}\right)<a^{\prime}, b^{\prime}<\alpha\left(y_{1}, y_{2}\right)$, and $a^{\prime}<$ $\beta\left(z_{1}, z_{2}\right)$ with $\alpha\left(z_{1}, z_{2}\right)<b^{\prime}$. This completes the proof of the theorem.

\section{Example}

In this section, as an application, the result is demonstrated with an example. 
Consider a coupled system of $p$-Laplacian fractional order BVP:

$$
\begin{gathered}
D_{0^{+}}^{1.7}\left(\phi_{p}\left(D_{0^{+}}^{2.6} u(t)\right)\right)=f_{1}(t, u(t), v(t)), \quad t \in(0,1), \\
D_{0^{+}}^{1.9}\left(\phi_{p}\left(D_{0^{+}}^{2.8} v(t)\right)\right)=f_{2}(t, u(t), v(t)), \quad t \in(0,1), \\
u(0)=D_{0^{+}}^{0.5} u(0)=0, \quad 5 u(1)+8 D_{0^{+}}^{0.7} u(1)=0, \\
D_{0^{+}}^{2.6} u(0)=D_{0^{+}}^{2.6} u(1)=0, \\
v(0)=D_{0^{+}}^{0.5} v(0)=0, \quad 5 v(1)+8 D_{0^{+}}^{0.7} v(1)=0, \\
D_{0^{+}}^{2.8} v(0)=D_{0^{+}}^{2.8} v(1)=0,
\end{gathered}
$$

where

$$
\begin{aligned}
& f_{1}(t, u, v)=\left\{\begin{aligned}
& \frac{e^{t}}{99}+\frac{\sin (u+v)}{10} \\
&+\frac{87(u+v)^{3}}{10}, 0 \leq u+v \leq 10, \\
& \frac{e^{t}}{99}+\frac{87000}{10} \\
&+\frac{\sin (u+v)}{10}, u+v>10 .
\end{aligned}\right. \\
& f_{2}(t, u, v)=\left\{\begin{array}{cc}
\frac{3 e^{t}}{159}+\frac{\cos (u+v)}{10} & 0 \leq u+v \leq 10, \\
+\frac{93(u+v)^{3}}{10}, & u+v>10, \\
\frac{3 e^{t}+\frac{93000}{159}}{+\frac{\cos (u+v)}{10},} &
\end{array}\right.
\end{aligned}
$$

Then the Green functions $G_{i}(t, s)$ and $H_{i}(t, s)$, for $i=1,2$, are given by

$$
\begin{aligned}
& G_{1}(t, s)=\left\{\begin{array}{cc}
\frac{1}{16.24}\left[\frac{5 \Gamma(1.9)}{\Gamma(2.6)}+8(1-s)^{-0.7}\right] & \\
\times[t(1-s)]^{1.6}, & t \leq s, \\
\frac{1}{16.24}\left[\frac{5 \Gamma(1.9)}{\Gamma(2.6)}+8(1-s)^{-0.7}\right] & \\
\times[t(1-s)]^{1.6}-\frac{(t-s)^{1.6}}{\Gamma(2.6)}, & s \leq t,
\end{array}\right. \\
& G_{2}(t, s)=\left\{\begin{array}{cc}
\frac{1}{18.64}\left[\frac{5 \Gamma(2.1)}{\Gamma(2.6)}+8(1-s)^{-0.7}\right] & \\
\times[t(1-s)]^{1.8}, & t \leq s, \\
\frac{1}{18.64}\left[\frac{5 \Gamma(2.1)}{\Gamma(2.6)}+8(1-s)^{-0.7}\right] & \\
\times[t(1-s)]^{1.8}-\frac{(t-s)^{1.8}}{\Gamma(2.8)}, & s \leq t,
\end{array}\right.
\end{aligned}
$$

$$
\begin{aligned}
& H_{1}(t, s)= \begin{cases}\frac{[t(1-s)]^{0.7}-(t-s)^{0.7}}{\Gamma(1.7)}, & s \leq t, \\
\frac{[t(1-s)]^{0.7}}{\Gamma(1.7)}, & t \leq s,\end{cases} \\
& H_{2}(t, s)= \begin{cases}\frac{[t(1-s)]^{0.9}-(t-s)^{0.9}}{\Gamma(1.9)}, & s \leq t, \\
\frac{[t(1-s)]^{0.9}}{\Gamma(1.9)}, & t \leq s .\end{cases}
\end{aligned}
$$

Clearly, the Green functions $G_{i}(t, s)$ and $H_{i}(t, s)$, for $i=1,2$, are positive and $f_{1}, f_{2}$ are continuous and increasing on $[0, \infty)$. By direct calculations, $\eta=0.08, p=2, L=33.16$, and $M=1677.73$. Choosing $a^{\prime}=1, b^{\prime}=10$ and $c^{\prime}=900$ and then $0<a^{\prime}<b^{\prime}<b^{\prime} / \eta \leq c^{\prime}$ and $f_{i}$, for $i=1,2$ satisfies

(i) $f_{i}(t, u, v)<16.5845=\phi_{p}\left(a^{\prime} L / 2\right), t \in[0,1]$ and $u, v \in$ $[0.08,1]$,

(ii) $f_{i}(t, u, v)>8388.65=\phi_{p}\left(b^{\prime} M / 2\right), t \in[1 / 4,3 / 4]$ and $u, v \in[10,125]$,

(iii) $f_{i}(t, u, v)<14926.09=\phi_{p}\left(c^{\prime} L / 2\right), t \in[0,1]$ and $u, v \in[0,900]$.

Then, all the conditions of Theorem 9 are satisfied. Therefore, it follows from Theorem 8 that the $p$-Laplacian fractional order BVP (51) has at least three positive solutions.

\section{Conflict of Interests}

The authors declare that there is no conflict of interests regarding the publication of this paper.

\section{Acknowledgment}

The authors thank the referees for their valuable comments and suggestions.

\section{References}

[1] R. W. Leggett and L. R. Williams, "Multiple positive fixed points of nonlinear operators on ordered Banach spaces," Indiana University Mathematics Journal, vol. 28, no. 4, pp. 673-688, 1979.

[2] R. P. Agarwal, D. O’Regan, and P. J. Y. Wong, Positive Solutions of Differential, Difference and Integral Equations, Kluwer Academic Publishers, Dordrecht, The Netherlands, 1999.

[3] D. R. Anderson and J. M. Davis, "Multiple solutions and eigenvalues for third-order right focal boundary value problems," Journal of Mathematical Analysis and Applications, vol. 267, no. 1, pp. 135-157, 2002.

[4] R. Avery and J. Henderson, "Existence of three positive pseudosymmetric solutions for a one-dimensional $p$-Laplacian," Journal of Mathematical Analysis and Applications, vol. 277, no. 2, pp. 395-404, 2003.

[5] C. Yang and J. Yan, "Positive solutions for third-order SturmLiouville boundary value problems with $p$-Laplacian," Computers \& Mathematics with Applications, vol. 59, no. 6, pp. 20592066, 2010. 
[6] R. P. Kapula, P. Murali, and K. Rajendrakumar, "Existence of positive solutions for higher order $(p, q)$-Laplacian two-point boundary value problems," International Journal of Differential Equations, vol. 2013, Article ID 743943, 9 pages, 2013.

[7] Z. Bai and H. Lü, "Positive solutions for boundary value problem of nonlinear fractional differential equation," Journal of Mathematical Analysis and Applications, vol. 311, no. 2, pp. 495-505, 2005.

[8] R. Dehghani and K. Ghanbari, "Triple positive solutions for boundary value problem of a nonlinear fractional differential equation," Bulletin of the Iranian Mathematical Society, vol. 33, no. 2, pp. 1-14, 2007.

[9] J. Henderson and S. K. Ntouyas, "Positive solutions for systems of nonlinear boundary value problems," Nonlinear Studies, vol. 15, no. 1, pp. 51-60, 2008.

[10] B. Ahmad and J. J. Nieto, "Existence results for a coupled system of nonlinear fractional differential equations with threepoint boundary conditions," Computers \& Mathematics with Applications, vol. 58, no. 9, pp. 1838-1843, 2009.

[11] X. Su, "Boundary value problem for a coupled system of nonlinear fractional differential equations," Applied Mathematics Letters, vol. 22, no. 1, pp. 64-69, 2009.

[12] C. Bai, "Existence of positive solutions for boundary value problems of fractional functional differential equations," Electronic Journal of Qualitative Theory of Differential Equations, vol. 2010, no. 30, pp. 1-14, 2010.

[13] C. S. Goodrich, "Existence of a positive solution to systems of differential equations of fractional order," Computers \& Mathematics with Applications, vol. 62, no. 3, pp. 1251-1268, 2011.

[14] N. Nyamoradi and M. Javidi, "Existence of multiple positive solutions for fractional differential inclusions with $m$-point boundary conditions and two fractional orders," Electronic Journal of Differential Equations, vol. 2012, no. 187, pp. 1-26, 2012.

[15] K. R. Prasad and B. M. B. Krushna, "Multiple positive solutions for a coupled system of Riemann-Liouville fractional order twopoint boundary value problems," Nonlinear Studies, vol. 20, no. 4, pp. 501-511, 2013.

[16] G. Chai, "Positive solutions for boundary value problem of fractional differential equation with p-Laplacian operator," Boundary Value Problems, vol. 2012, article 18, 20 pages, 2012.

[17] R. I. Avery, "A generalization of the Leggett-Williams fixed point theorem," Mathematical Sciences Research Hot-Line, vol. 3, no. 7, pp. 9-14, 1999. 


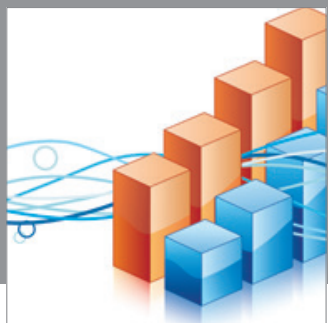

Advances in

Operations Research

mansans

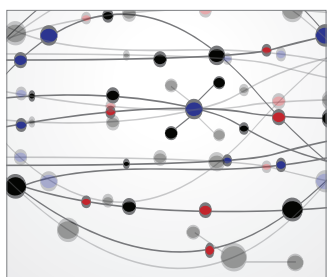

The Scientific World Journal
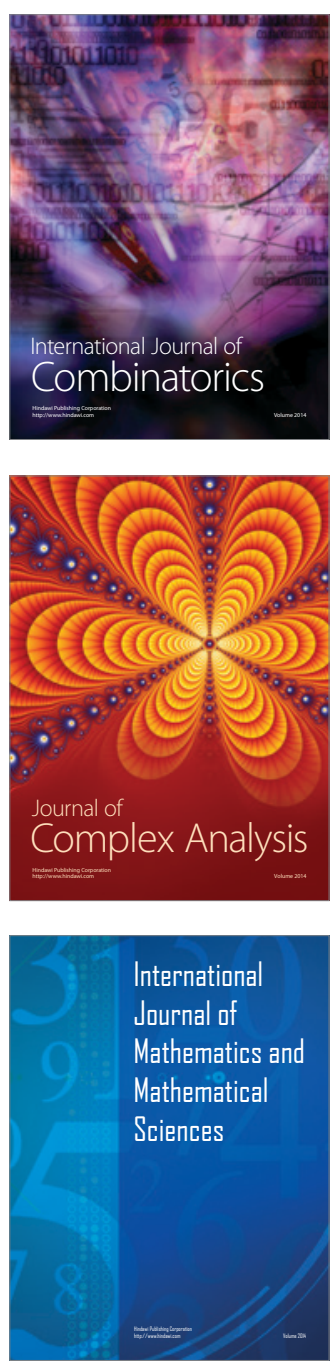
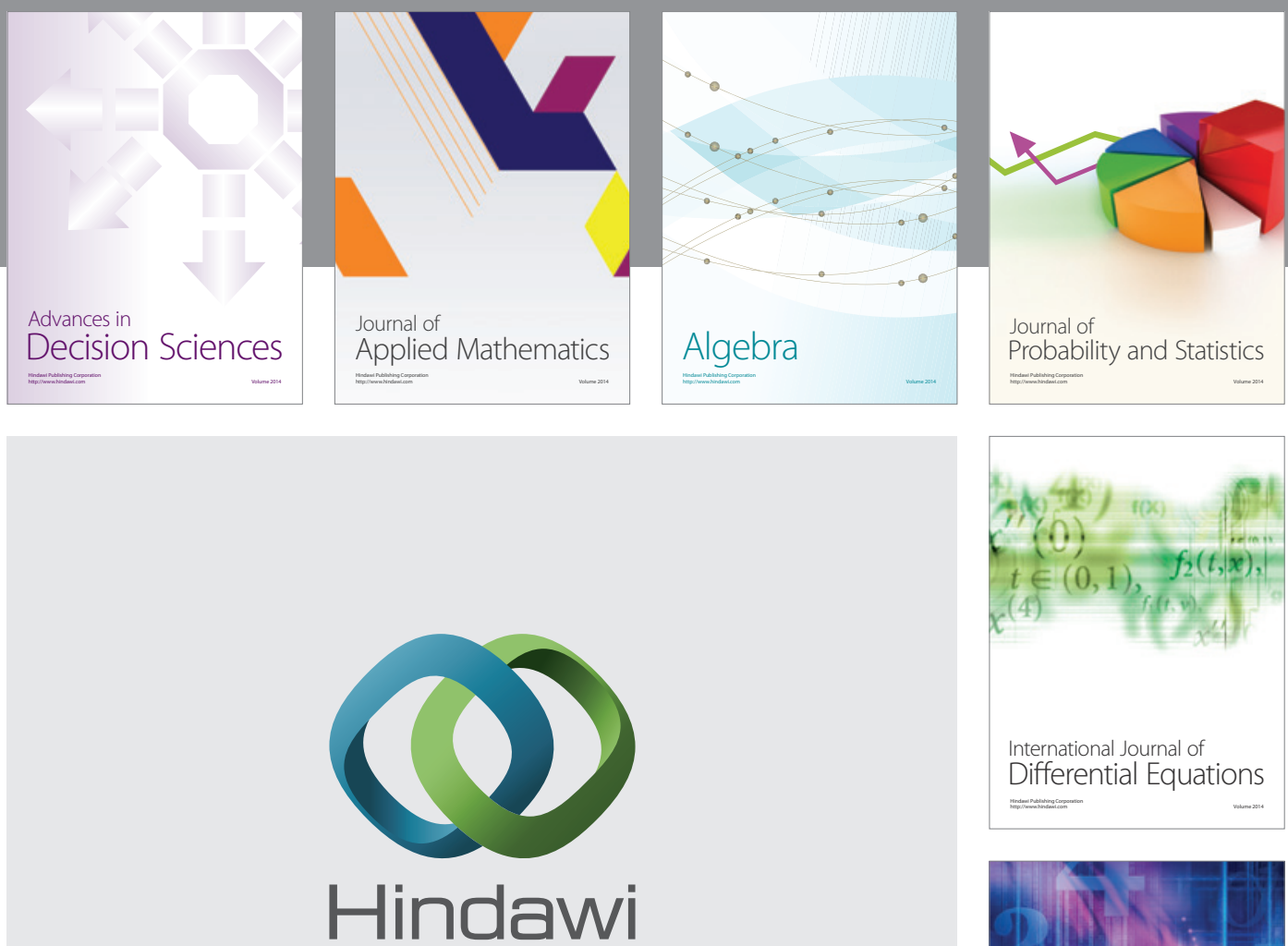

Submit your manuscripts at http://www.hindawi.com
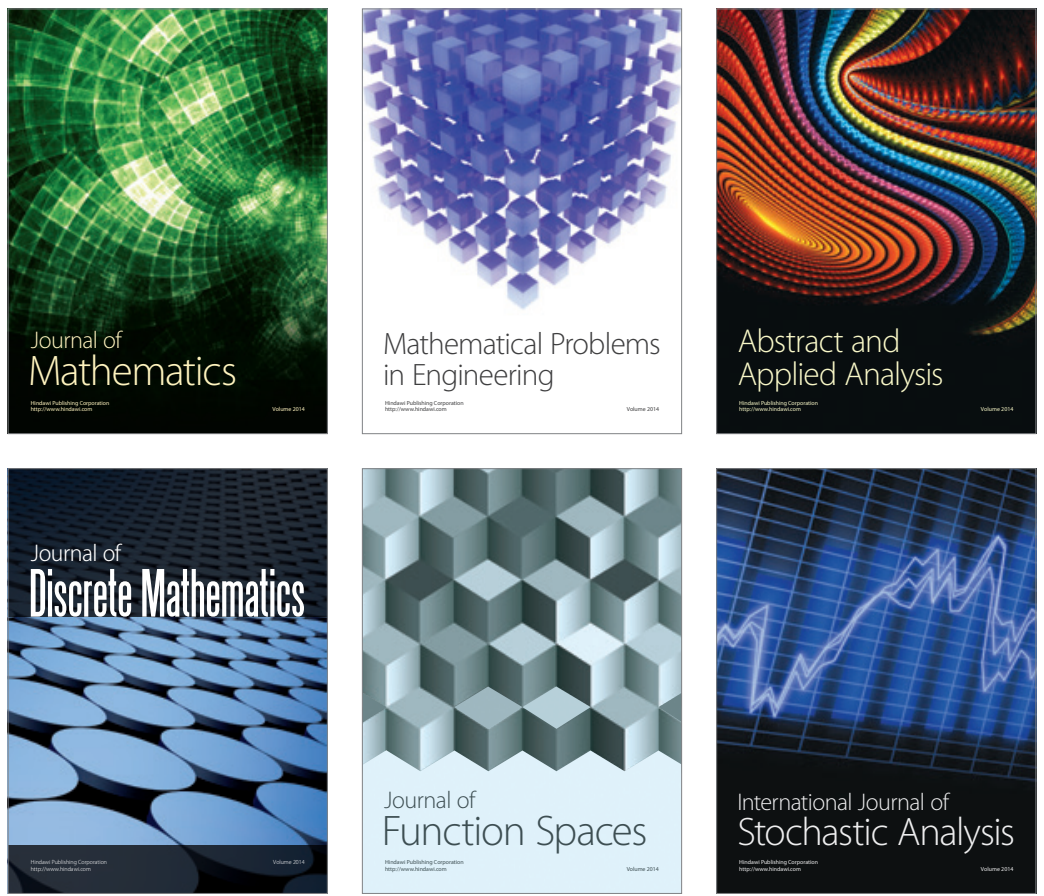

Journal of

Function Spaces

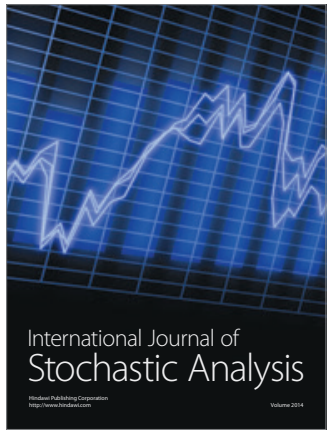

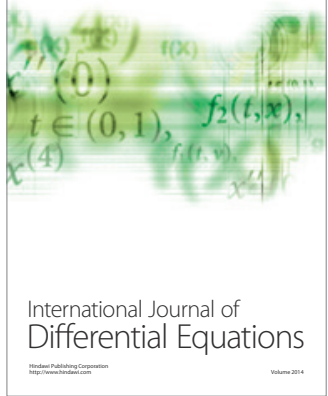
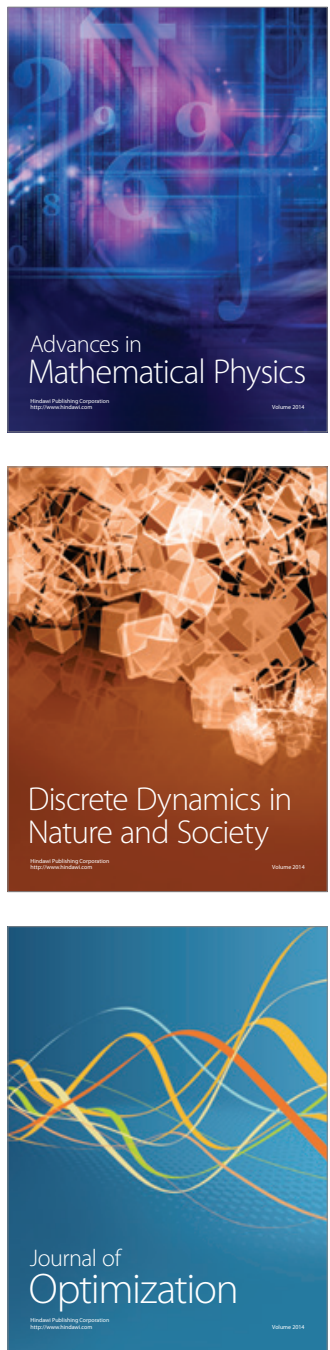